\title{
CONCENTRACIÓN DE LA RIQUEZA EN AmÉRICA LATINA EN EL SIGLO XXI
}

\author{
Germán Alarco Tosoni ${ }^{a}$ y César Castillo García ${ }^{b}$
}

Fecha de recepción: 6 de enero de 2020. Fecha de aceptación: 5 de mayo de 2020.

$$
\text { http://doi.org/10.22201/iiec.20078951e.2020.203.69534 }
$$

\begin{abstract}
Resumen. El artículo presenta la magnitud de la riqueza de las diferentes economías de América Latina y el proceso de concentración de ésta hacia los billonarios (superricos) y los tramos más altos en perjuicio de los pequeños ricos, las clases medias-altas y medias -a excepción de Chile, Perú y otras economías-. Si bien América Latina tiene una elevada concentración de la riqueza, su coeficiente Gini es menor al del resto de las regiones del mundo. En el periodo de estudio se registra una clara tendencia creciente en la concentración de la riqueza de la región y el mundo.
\end{abstract}

Palabras clave: distribución de la riqueza; concentración; riqueza productiva; desigualdad; coeficiente de Gini; billionarios.

Clasificación JEL: D31; D33; E25; N36.

\section{Concentration of Wealth in Latin AMERICA IN THE 2 IST CENTURY}

\begin{abstract}
The article illustrates the degree of wealth in various Latin American economies and the process of wealth concentration towards billionaires (super-rich) and the highest economic strata to the detriment of the affluent, the upper-middle classes, and the middle classes -with the exception of Chile, Peru, and several other economies. Although Latin America has a high concentration of wealth, its Gini coefficient is lower than that of other regions of the world. A clear upward trend in the concentration of wealth in the region and the world can be seen throughout the period analyzed.
\end{abstract}

Key Words: distribution of wealth; concentration; productive wealth; inequality; Gini coefficient; billionaires.

\footnotetext{
a Escuela de Postgrado y miembro del Centro de Investigación de la Universidad del Pacífico, Perú; ${ }^{b}$ Universidad del Pacífico, Perú. Correos electrónicos: g.alarcotosoni@up.edu.pe y c.castillog@up.edu.pe, respectivamente. Los autores agradecen el valioso apoyo de Martín Astocondor asistente de investigación, así como los comentarios de Patricia del Hierro.
} 


\section{INTRODUCCIÓN}

Hoy en día la elevada desigualdad económica es una preocupación internacional. Incluso en el Foro Económico Mundial (2018), que reúne a grandes empresarios y gobiernos del mundo, se señala como una de las tendencias y riesgos principales de la economía mundial. El incremento en las disparidades de la riqueza e ingresos comparte prioridad al igual que el cambio climático, el envejecimiento poblacional y el incremento de la polarización de las sociedades, entre otros temas, pues sus impactos son económicos, sociales y políticos en el corto, mediano y largo plazos. En otras palabras, el nivel y la variabilidad de la actividad económica se afectan negativamente (Ostry et al., 2014), se agudizan los conflictos sociales y aumentan las pretensiones de los grupos de poder económico respecto a condicionar las políticas de acuerdo a sus intereses particulares (OXFAM, 2016).

Hay discusión y algunas coincidencias sobre la evolución de la desigualdad global y al interior de las economías. Bourguignon [2017 (2012)] y Milanovic [2017 (2016)] plantean a nivel global que desde el siglo XIX existe una tendencia ascendente respecto a la concentración del ingreso con una desaceleración y caída entre los años cincuenta y setenta; luego continúa la tendencia creciente. La discusión se presenta a partir de los ańos noventa dado el mayor crecimiento de las clases medias de las economías emergentes. Sin embargo, cuando se revisa la información al interior de los países queda claro que la distribución funcional del ingreso a favor del capital elevó su participación respecto de la cuota de los salarios de los años ochenta en adelante. Lo mismo ocurrió a favor de 10 o $1 \%$ más rico de la población (Piketty y Saez, 2003; OCDE, 2008; Piketty, 2014; CEPAL, 2018).

América Latina no resulta ajena a esta polémica. ${ }^{1}$ La distribución factorial del ingreso muestra una trayectoria sinusoidal en lo relativo a la cuota de los salarios y de las ganancias (Alarco, 2014 y 2017), donde a pesar de las particularidades nacionales el pico de la participación de los salarios se obtuvo en la edad de oro del capitalismo (los años sesenta). Se tienen mejoras en los últimos años, pero se explican más por el peso de las políticas implantadas sobre todo en Brasil y Argentina. Sin embargo, a partir de lo observado por Lustig y López-Calva (2010), entre otros, el Gini de la distribución personal del ingreso se redujo concluyendo que las reformas estructurales implantadas desde la

1 Trueba y Remuzgo (2016) realizan un recuento de autores que explican la desigualdad en América Latina; mientras que Alvaredo y Gasparini (2015) presentan una síntesis de la información sobre distribución del ingreso para las economías en desarrollo. 
década de los noventa resultaron exitosas. Sin embargo, se olvidan de que las encuestas de ingresos de los hogares omiten a los sectores de altos ingresos (y medios altos), a las ganancias del capital extranjero y a los crecientes procesos de fusiones y adquisiciones que concentran la propiedad del capital en pocas manos. ${ }^{2}$ Solimano (2016) también critica estas evaluaciones optimistas con información sobre los grupos de altos ingresos, distribución de la riqueza y datos tributarios que reflejan mayores niveles de desigualdad. ${ }^{3}$

En la tradición de los economistas clásicos, el análisis de la distribución de la riqueza, en particular de la propiedad de los medios de producción o riqueza productiva, es un factor clave para explicar la distribución funcional del ingreso entre capital o trabajo. La riqueza se define como el total de activos productivos y financieros, propiedad de las personas físicas o naturales en una economía cualquiera; asimismo, cuando se trata de la riqueza neta se deducen las deudas o pasivos de esas personas. La posesión de activos productivos determina la existencia de ingresos o rentas a favor del capital o, al contrario, si se trata de ingresos por conceptos de remuneraciones (sueldos y salarios) o ingresos mixtos (por trabajo independiente). La distribución personal del ingreso está a su vez condicionada por la cadena de elementos anteriores afectada por un conjunto de factores estructurales y económicos (nacionales e internacionales), sociales, políticos, institucionales y personales. La política fiscal (tributaria y de gasto social) es una de las que impacta significativamente sobre los resultados distributivos (Baéz Melián, 2018).

El presente texto evalúa la magnitud y concentración de la riqueza productiva en América Latina a través de la información obtenida por el Banco Mundial y estimaciones independientes elaboradas por Credit Suisse, Forbes y Knigth Frank. A diferencia de lo que ocurre en las economías desarrolladas, se acude a fuentes independientes, ya que desafortunadamente en la región no existen estadísticas oficiales derivadas de encuestas de activos y deuda de los hogares, registros de impuestos patrimoniales y de las herencias e información sobre ingresos por inversiones.

El artículo pretende responder a las siguientes interrogantes: ¡cuál es el valor de la riqueza productiva en las principales economías de América Latina?, ¿cuál es la importancia de los billonarios en la región?, ¿cómo se dis-

2 En Alarco (2018) se evalúan los efectos redistributivos, sobre la demanda y el crecimiento económico de los procesos de fusiones y adquisiciones en América Latina.

3 Alarco et al. (2019) evalúan la información oficial sobre la desigualdad elaborada a partir de las encuestas de ingreso gasto de los hogares, presentan diversos estudios críticos y corrigen resultados para Perú. 
tribuye y concentra la riqueza bajo diferentes estimaciones?, ¿qué ocurre con la desigualdad de esa riqueza intra, interregional y a través del tiempo? El artículo tiene cuatro secciones más la introducción y las conclusiones. En la primera, se presentan tanto los criterios de medición de la riqueza como las diferentes fuentes de información. En la segunda sección se muestran diversas mediciones absolutas de la riqueza para las diferentes economías de América Latina. La tercera sección presenta la información de los billonarios de Forbes; las estructuras de distribución de la riqueza más detalladas de Credit Suisse y Knight Frank. Por último, se observa la comparación interregional e intrarregional. ${ }^{4}$

\section{MEDICIÓN DE LA RIQUEZA, FUENTES DE INFORMACIÓN Y METODOLOGÍAS}

\section{Criterios y evolución general}

La contabilidad de la riqueza es una tarea compleja dado que la mayoría de los sistemas de información estadística no realizan un seguimiento pormenorizado de las cuentas de acervo o stock del capital para las economías nacionales. Un primer intento de medición de la riqueza a precios constantes se presenta en Goldsmith (1962) en el que se parte de los flujos de inversión bruta, inversión neta y los gastos en depreciación para cada tipo de activo considerado en el acervo nacional de Estados Unidos. Los acervos estimados consideran la vida útil de los activos y su valor se descuenta por el coeficiente de depreciación estimado para cada uno de ellos siguiendo la fórmula del inventario perpetuo.

Davies y Shorrocks (2000) utilizan un enfoque microeconómico para medir la magnitud de la riqueza y determinar su distribución basada en la información que se puede obtener de individuos y hogares, y se documentan fuentes de datos diferentes. El primer tipo de información se obtiene de las encuestas de hogares en las que se registran las tenencias de activos y deuda. Sin embargo, se hace menos confiable debido a los errores de muestreo o de reporte (algunos hogares no están capacitados, rechazan responder, subreportan la propiedad de ciertos activos o subvalúan los mismos). La segunda es la data impositiva sobre riqueza y patrimonio que se deriva de los registros de impuesto a la riqueza o que se obtiene de la construcción de multiplicadores

4 Las fuentes de información independientes sobre la riqueza son relativamente recientes (a excepción de Forbes), razón por la cual los periodos de análisis de este artículo son cortos. 
relacionados con el impuesto al patrimonio. Algunos de los problemas de la información de impuestos a la riqueza es que puede dejar de considerar algún tipo de acervo de riqueza (por ejemplo, bienes de consumo duraderos) o brindar información subvaluada de algunos activos. Asimismo, la información del impuesto a la propiedad puede estar subestimada porque no incluye a la población con propiedades por debajo del umbral de cobro de impuestos o bien omite la porción de la riqueza que se acoge a exoneraciones. ${ }^{5}$

La riqueza medida utilizando los datos sobre ingreso de capital se construye mediante el método de capitalización del ingreso (Giffen, 1913). Si se cuenta con información del impuesto a la renta, se puede dividir la información del ingreso por inversión sobre la participación del impuesto por ganancias del capital correspondiente. Finalmente, se pueden utilizar estadísticas publicadas sobre las tenencias de riqueza o activos de ciertos individuos o familias (información que se puede encontrar en bases de datos como las de Forbes o Fortune).

Davies et al. (2010) construyen hojas de balance con información de la riqueza financiera y no financiera para los hogares con un enfoque macroeconómico. Su trabajo abarcó 19 países, en su mayoría de altos ingresos y otros clasificados como de ingresos medios altos. Para incluir estadísticas de regiones como Asia, África y América Latina y el Caribe se complementó con la construcción de tablas con datos que aparecen en las encuestas de hogares. Estos autores encuentran que la mayor riqueza de estos países está conformada por activos no financieros. Al tiempo que sostienen que activos como terrenos y granjas son más comunes en los países menos desarrollados, mientras que los activos financieros son más importantes en los países ricos. En economías en vías de desarrollo, los activos financieros son principalmente cuentas de ahorro, mientras que en los países más ricos son las tenencias de acciones.

Roine y Waldenström (2015) discuten el concepto de riqueza a partir de la revisión de literatura empírica que incluye la construcción de series de largo plazo. El concepto de riqueza neta consiste en la suma de los valores de los activos reales y financieros menos la deuda. El primer tipo incluye propiedades, terrenos, bienes de consumo duradero y algunos bienes de lujo. Los activos financieros incluyen efectivo, depósitos bancarios, cuentas de seguro, depósitos de pensiones, acciones, bonos y otro tipo de tenencias bursátiles. Las deudas aglomeran la suma de hipotecas y créditos al consumo, la inversión y la educación. Al valorizarse a precios de mercado, el valor de la riqueza neta se

5 En Argentina, Colombia, Chile y Uruguay existe información consolidada sobre los ingresos e impuestos de las personas más ricas a partir de las declaraciones fiscales (Jiménez, 2015). 
hace muy sensible a las fluctuaciones cotidianas. Las mediciones no incluyen las cuentas de pensiones ni los valores de los bienes de consumo durable por problemas de reporte o por dificultades de valorización. Los resultados para diferentes países europeos y Estados Unidos cubren el periodo 1740-2011.

Saez y Zucman (2016) reconstruyen la riqueza total de los hogares en Estados Unidos en dos pasos: el periodo posterior a 1945 según las hojas de balance de hogares tomada de las cuentas financieras de Estados Unidos y el periodo de 1913-1945 a partir de distintos trabajos de reconstrucción basados en dichas cuentas. Tras obtener dicho agregado se identifica la distribución del ingreso por capital a partir de los registros de impuestos de individuos obtenidos de fuentes oficiales o de distintas investigaciones. Por su parte, Alvaredo et al. (2019) presentan resultados de medición de la riqueza y su distribución para economías como Estados Unidos, Francia, España, Reino Unido utilizando una combinación de encuestas de riqueza de los hogares, impuestos sobre ingreso que utilizan el método de capitalización del ingreso y datos de impuestos a las herencias que emplean también un multiplicador de herencia. Asimismo, se tienen estimados para Alemania y Suecia, entre otras economías, que se comentan en Piketty (2019). En tanto, De Rosa (2019) utilizando las metodologías anteriores acaba de presentar resultados agregados para Uruguay.

\section{Metodologías y fuentes de información}

La información para este estudio proviene sobre todo de cuatro fuentes: Banco Mundial, Credit Suisse, Forbes y Knight Frank. La primera fuente registra información relativa al valor de la riqueza e información macroeconómica diversa. Para el Banco Mundial (2018a), el capital productivo se entiende como los activos fabricados o construidos, como maquinaria, equipos y estructuras físicas, incluyendo el valor del suelo urbano. ${ }^{6}$ La estimación del acervo de capital se realiza a través del método del inventario perpetuo adoptado por la mayoría de los países de la Organización para la Cooperación y el Desarrollo Económicos (OCDE). Este organismo internacional aprovecha la información de la base de datos de Penn World Table 9,0 que utilizan esta metodología para estimar el stock de capital en 172 países desde 1970 hasta 2014.

Credit Suisse define la riqueza como el patrimonio neto que es el valor comercial de los activos financieros más los activos no financieros menos deudas.

6 No se considera aquí la valorización del capital natural, tierras agrícolas, capital humano y activos externos netos. Así como tampoco se contemplan bienes de consumo duradero y propiedades de uso personal. 
Los activos financieros consisten en efectivo, depósitos bancarios y acciones. Uno de los inconvenientes para calcular la riqueza, es que ningún país tiene una única fuente completa de información sobre la riqueza personal. Por ejemplo, los países de ingresos medios y bajos tienen poca data. La metodología es esencialmente un enfoque de abajo hacia arriba, comienza estableciendo el nivel promedio de riqueza en los diferentes países en los que se incluye el patrón de tenencia de riqueza derivado de las encuestas de hogares y otras fuentes. ${ }^{7}$ Por los problemas de subestimación se requieren de ajustes para construir estimaciones del número de individuos ricos y del tamaño de sus tenencias. La cola superior de la distribución de la riqueza suele ser aproximada mediante la distribución de Pareto. ${ }^{8}$ Además, para determinar la forma precisa de la cola de la riqueza superior se utiliza la lista proporcionada por Forbes.

Por otra parte, Knight Frank es más escueto. Al igual que la fuente anterior, incluye los activos financieros y no financieros menos deuda. Asimismo, su principal fuente de información es directa, ya que se basa en las respuestas de casi 900 de los principales banqueros privados y asesores de riqueza del mundo, que representan a más de 10000 clientes con una riqueza combinada de alrededor de US $\$ 2$ billones. New World Wealth' le proporciona la distribución de riqueza, muestra su crecimiento histórico y previsto en cinco rangos a nivel regional, nacional y de principales ciudades.

\section{MEDICIONES ABSOLUTAS}

Se presentan diversas estimaciones de la riqueza elaboradas tanto por el Banco Mundial como por Credit Suisse. Los resultados son diferentes en razón a las metodologías consideradas y a los diversos periodos bajo análisis de cada fuente: 2005 y 2014 en un caso y 2010, 2012 y 2016 en otro. En el cuadro 1 se muestra también el PIB de 2014 y la relación capital-producto obtenido

7 En el caso que no se disponga de encuestas de los hogares que incluyan reportes sobre activos y deudas se realizan estimaciones de regresión empleando variables correlacionadas de diferentes economías. En última instancia se utilizan promedios de las economías de los mismos niveles de ingreso ubicadas en esa región. Otra dificultad es que algunos países tienen observaciones directas sobre la riqueza financiera, pero requieren que se calcule el valor de la riqueza no financiera. En el caso que no tuvieran actualizada esta información se ajusta con los índices de precios y cotizaciones de los mercados de valores específicos, índices de precios al consumidor o bien otros componentes del Producto Interno Bruto (РІв).

8 Se trata de una función de distribución continua.

9 New World Wealth es un grupo de investigación con sede en Johannesburgo, Sudáfrica, especializado en encuestas, informes de países y estadísticas de riqueza. Su investigación abarca 90 países y 150 ciudades en todo el mundo <http://www.newworldwealth.com/> 
como cociente de la riqueza productiva de ambas fuentes estadísticas entre el producto.

De acuerdo a la información del Banco Mundial para 2014 la economía con mayores niveles de riqueza era Brasil, seguida de México y Argentina. A nivel intermedio se ubicó Venezuela, Colombia, Chile y Perú; mientras el resto de economías tuvo un valor de riqueza más pequeño asociado a su menor importancia. Por otra parte, debe anotarse que las estimaciones del Credit Suisse se encontrarían en todos los casos por debajo de las realizadas por el Banco Mundial con menores diferencias en los casos Chile, Costa Rica, El Salvador y Perú. Las razones para explicar estas diferencias pueden ser diversas.

Cuadro 1. Estimaciones de la riqueza en diferentes economías de América Latina (US\$ millones)

\begin{tabular}{|c|c|c|c|c|c|c|c|}
\hline \multirow[t]{2}{*}{ Economías } & \multicolumn{4}{|c|}{ Banco Mundial } & \multicolumn{3}{|c|}{ Credit Suisse } \\
\hline & 2005 & 2014 & PIB 2014 & Capital/PIB & 2010 & 2012 & 2016 \\
\hline Argentina & 419050 & 1627611 & 526320 & 3.09 & 469645 & 491567 & 467354 \\
\hline Bolivia & 18364 & 69983 & 32996 & 2.12 & 17313 & 24315 & 34222 \\
\hline Brasil & 2111968 & 6608300 & 2455994 & 2.69 & 3263519 & 3280533 & 2537416 \\
\hline Chile & 313974 & 801024 & 260584 & 3.07 & 297244 & 541646 & 602173 \\
\hline Colombia & 320329 & 1331325 & 378196 & 3.52 & 514952 & 748085 & 609333 \\
\hline Costa Rica & 46401 & 117422 & 50578 & 2.32 & 58787 & 82684 & 105032 \\
\hline $\begin{array}{l}\text { República } \\
\text { Dominicana }\end{array}$ & 76145 & 226933 & 66065 & 3.43 & n.d. & n.d. & n.d. \\
\hline Ecuador & 100549 & 325517 & 101726 & 3.20 & 100013 & 98746 & 125904 \\
\hline El Salvador & 35788 & 62396 & 22586 & 2.76 & 37719 & 41801 & 55266 \\
\hline Guatemala & 67657 & 153028 & 58722 & 2.61 & n.d. & n.d. & n.d. \\
\hline Honduras & 29828 & 67093 & 19756 & 3.40 & n.d. & n.d. & n.d. \\
\hline México & 2197860 & 5005152 & 1314385 & 3.81 & 1759846 & 2150610 & 1621280 \\
\hline Nicaragua & 16102 & 54576 & 11880 & 4.59 & 9079 & 11435 & 12408 \\
\hline Panamá & 37718 & 117488 & 49921 & 2.35 & 36132 & 49776 & 57141 \\
\hline Paraguay & n.d. & 77765 & 30881 & 2.52 & 26238 & 37056 & 42277 \\
\hline Perú & 200253 & 604658 & 201081 & 3.01 & 262990 & 325345 & 520450 \\
\hline Uruguay & 32208 & 219700 & 57236 & 3.84 & 89003 & 107157 & 89685 \\
\hline
\end{tabular}


Cuadro 1. Estimaciones de la riqueza en diferentes economías de América Latina (US\$ millones) (continuación)

\begin{tabular}{lrrrrrrrr}
\hline Economías & \multicolumn{4}{c}{ Banco Mundial } & \multicolumn{4}{c}{ Credit Suisse } \\
\cline { 2 - 5 } & \multicolumn{1}{c}{2005} & 2014 & PIB 2014 & Capital/PIB & 2010 & 2012 & \multicolumn{1}{c}{2016} \\
\hline Venezuela & 421591 & 2153203 & 482359 & 4.46 & & 279089 & 184576 & 84416 \\
América Latina & 6445784 & 19623174 & 6121267 & 3.21 & & 7221569 & 8175334 & 6964358 \\
\hline
\end{tabular}

Notas: n.d.: no determinado.

Fuente: elaboración propia con base en Banco Mundial (2011 y 2018a) y Credit Suisse (2011, 2013 y 2017).

\section{DISTRIBUCIÓN DE LA RIQUEZA}

\section{Billionarios latinoamericanos}

El número de billionarios ${ }^{10}$ de América Latina ha crecido a lo largo del tiempo. En el cuadro 2 se observa la información de Forbes para algunos ańos seleccionados. Entre 2009 y 2016 pasó de 36 a 87 latinoamericanos con una riqueza igual o superior al monto señalado. La economía con el mayor número fue Brasil, seguido por México, Chile, Argentina, Perú, Colombia y Venezuela. El resto de economías de la región no tienen billonarios. Debe notarse que entre 2014 y 2016 se redujo el número de billonarios en Brasil y México debido a la mayor depreciación de la moneda local. En términos absolutos, la mayor riqueza de los superricos está en Brasil con US\$172 100 millones, seguido por México con US\$116 700 millones y Chile con US\$41 400 millones; en menor medida en Venezuela con US\$5 100 millones y Perú con US\$8 400 millones. En Argentina estos patrimonios netos suman US $\$ 12400$ millones.

Los 87 billonarios de la región en 2016 tienen un patrimonio neto de US\$373 200 millones superior al pIB nominal de Venezuela, Colombia o Perú. En términos relativos, los superricos de Chile tienen un patrimonio neto equivalente a $16.6 \%$ del PIB chileno, seguidos por los mexicanos con un monto equivalente a $10.9 \%$ del PIB del país y después de los brasileños con 9.6\% del рів nacional. Todos los billonarios de la región tienen patrimonios netos equivalentes a $8.3 \%$ del PIB regional. Cuando estos patrimonios netos se expresan respecto de la riqueza productiva reportada por el Banco Mundial para 2014, los billonarios de la región tienen $2.1 \%$ de la riqueza productiva total, con un mayor valor en Chile (5.2\%), Brasil (2.6\%) y México (2.3\%).

10 En la nomenclatura norteamericana cuentan con un patrimonio neto de más de US\$1 000 millones. 


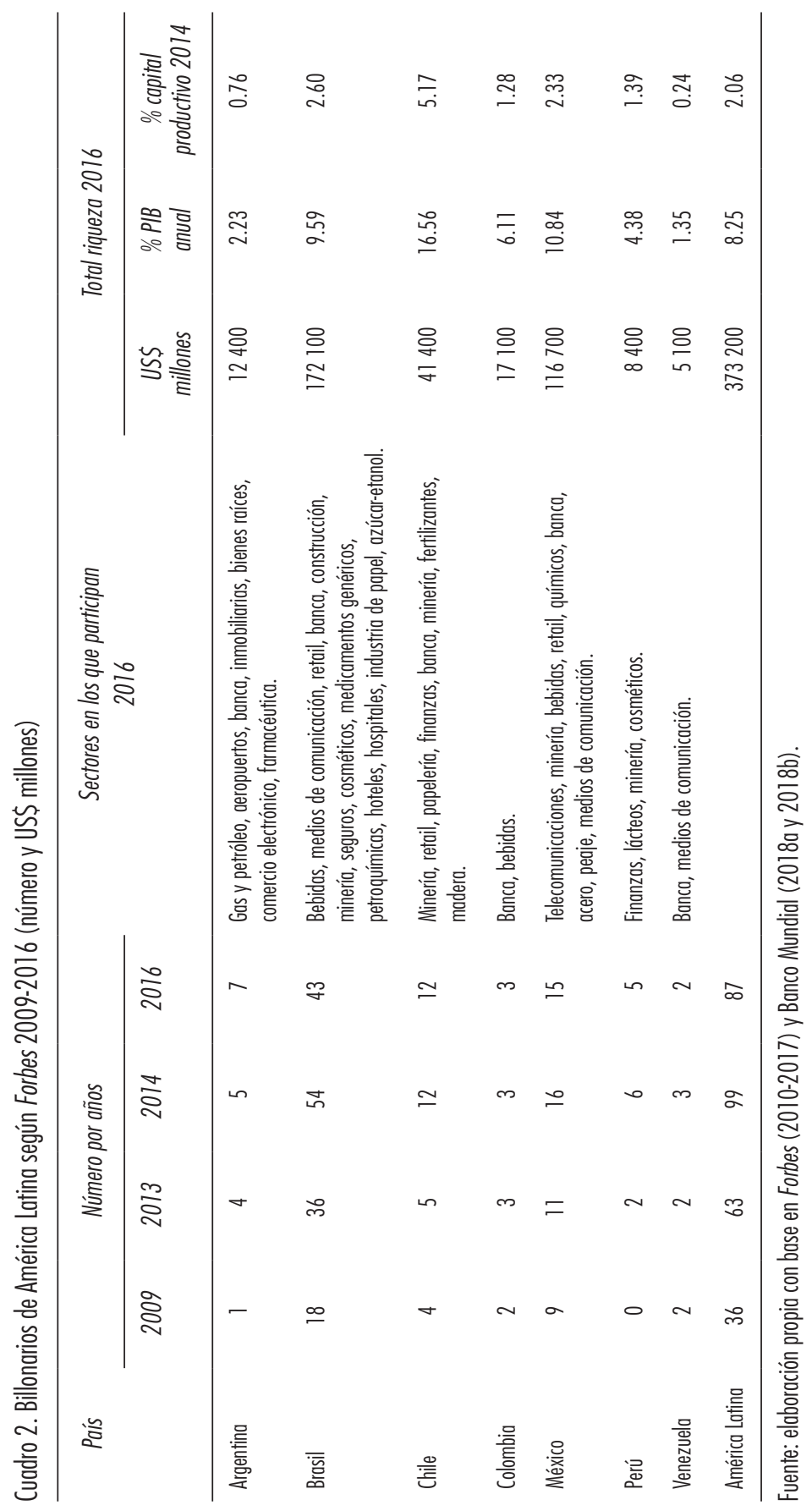


La importancia de los billionarios tanto a nivel mundial como América Latina se muestra en la gráfica 1, en la que se presenta la participación porcentual de la riqueza de los billonarios del mundo entre el piB mundial y de la riqueza de los billonarios de los países de la región América Latina entre su respectivo PIB para el periodo 1999-2016. Ambos cocientes permitirían evaluar la tendencia en la concentración de la riqueza en manos de los superricos en los dos ámbitos analizados.

La tendencia en la participación de los billionarios del mundo sigue la línea de lo planteado por Milanovic [2017 (2016)] en que esta tendencia es creciente en el tiempo. En 1999 representaban poco menos de 4.3\% del PIB mundial, mientras que en 2016 su participación era ligeramente superior a $10.1 \%$ del PIB anual. Esta misma tendencia se observa en el caso de los billonarios de la región. En 1999 tenían una participación equivalente a 3.5\% del PIB, para mostrar en 2016 una cuota de $8.3 \%$ del рів. En lo que va del siglo Xxi se muestra un proceso claro de mayor concentración de la riqueza en manos de los más ricos del mundo.

Gráfica 1. Tendencia de la participación de los ingresos de los billonarios de América Latina y el mundo respecto del PIB (\%)

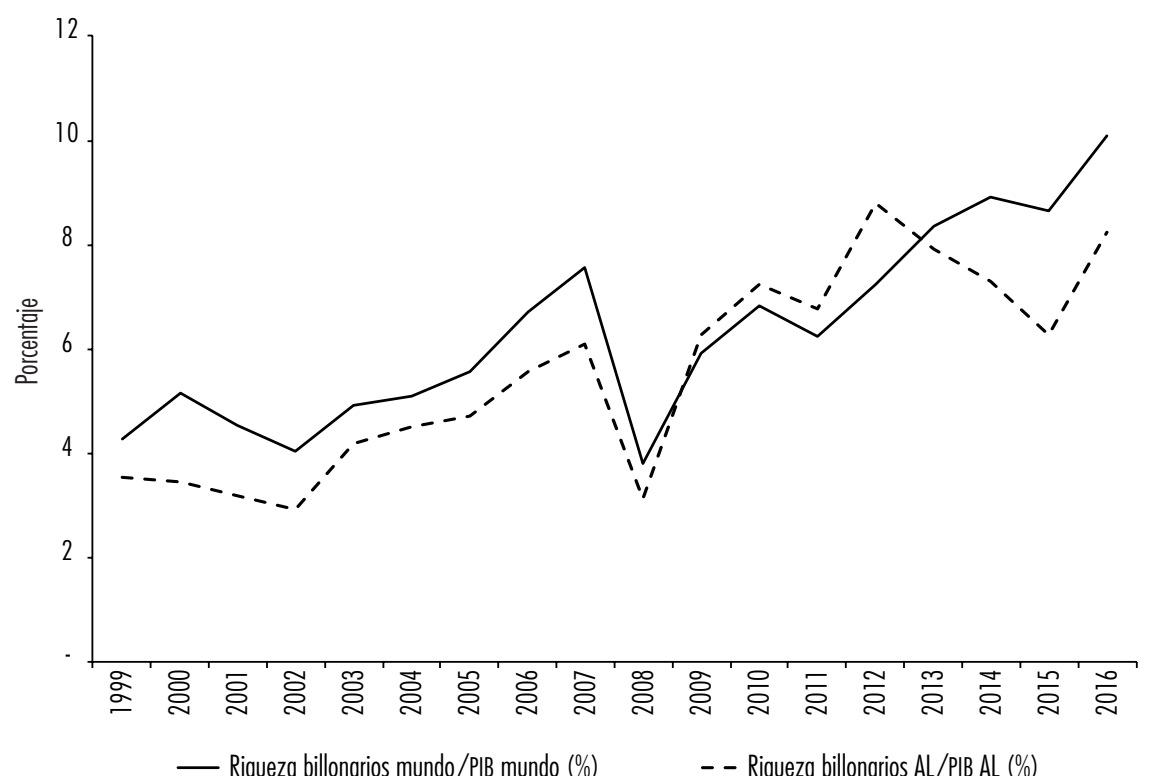

Fuente: elaboración propia con base en Forbes (2010-2017) y Banco Mundial (2018a y 2018b). 


\section{Concentración por estratos}

En los cuadros 3 y 4 se presenta la estructura de distribución de la riqueza de las diferentes economías de la región; la diferencia reside en que en el primer cuadro se detallan 10 rangos para 6 economías de la región, mientras que en el segundo, sólo se distingue entre quienes tienen más de US\$1 millón y los rangos inferiores para 13 economías de la región. Esta fuente de información no muestra mayores detalles de los que aquí se presentan. En términos absolutos, las economías con mayor número de millonarios son Brasil, México, Chile, Perú, Colombia y Argentina.

Las personas que cuentan con una riqueza superior a US\$100 millones han pasado de 1108 personas en 2012 a 1494 personas en 2016 con una tasa superior a $7.8 \%$ anual, mayor al crecimiento del PIB regional de $0.8 \%$ anual. Aún entre los que cuentan con un patrimonio neto entre US\$50 y 100 millones pasaron a ser 1985 personas en 2016 respecto de 1667 personas en 2012 con una tasa de crecimiento promedio anual de 4.5\%. Sin embargo, la situación del conjunto de economías analizadas es estacionaria entre los que tienen US\$5 y 50 millones; mientras que el número de millonarios entre US $\$ 1$ y 5 millones se ha reducido en Argentina, Brasil, Colombia y México por el menor valor de mercado de sus patrimonios netos. Sólo en Chile y Perú se observa un crecimiento significativo del número de millonarios en el rango entre US\$1 y 5 millones.

Tanto en los cuadros 3 y 4 con 6 y 13 economías, respectivamente, queda claro que el número de adultos con patrimonios netos entre US\$10 $000 \mathrm{y}$ 1 millón se ha reducido para los dos conjuntos de economías seleccionadas con lo que se vislumbraría una caída en los patrimonios netos de las clases medias y medias y medias-altas. Por el contrario, el número de adultos con un patrimonio neto menor a US\$10 000 se ha incrementado significativamente en toda la región. Las únicas economías donde se ha incrementado el número de adultos con patrimonio neto entre US\$100 000 y 1 millón son Bolivia, Ecuador, El Salvador, Nicaragua, Paraguay y Perú. Asimismo, en el periodo bajo análisis se ha elevado el número de adultos con un patrimonio neto entre US\$10 000 y 100000 en Argentina, Bolivia, Colombia, Ecuador, El Salvador, Perú y Uruguay.

En tanto, en el cuadro 5 se muestra la proporción de la riqueza en manos de la población más rica de cuatro economías de América Latina: Brasil, Chile, Colombia y México y los grandes grupos regionales. Se detalla la participación en la riqueza total del 10, 5 y 1\% de la población más rica. La data es de Credit Suisse para 2015 y 2016. No hay información para otras economías de la región ni otros periodos de tiempo previos. $\mathrm{Al}$ respecto, se aprecia una 
mayor concentración en Brasil y Chile, seguida de México y Colombia. En el promedio regional el $1 \%$ más rico tiene $42 \%$ de la riqueza y el $10 \%$ superior tienen $71.2 \%$ de la riqueza total. Con estos resultados América Latina se ubica a un nivel intermedio respecto a la concentración de la riqueza por encima de Europa, Asia Pacífico y ligeramente por arriba de América del Norte. Sin embargo, América Latina tiene una menor concentración en el percentil más rico que India, África y China. ${ }^{11}$

La información proporcionada por Knight Frank comprende sólo 10 economías, 3 rangos y 1 año similar al de Credit Suisse (véase cuadro 6). No obstante, se observan tendencias comunes. En primer lugar, el número personas con un patrimonio neto mayor a US $\$ 100$ millones se eleva significativamente entre 2005 y 2016 al pasar de 588 a 1006 personas. En segundo lugar, este fenómeno también se presenta entre los que tienen US\$30 millones y $100 \mathrm{mi}-$ llones y en menor medida entre las personas que poseen un patrimonio neto entre US\$10 millones y 30 millones. En tercer lugar, el número de personas con un patrimonio neto mayor a US\$1 millón pero menor a 10 millones aumentó respecto del año base 2005. Sin embargo, en Brasil, Colombia, México y Venezuela se redujeron los valores totales de los patrimonios netos entre 2015 y 2016.

En el cuadro 7 se presenta la participación de los millonarios respecto del total de la población de las respectivas economías para 2016 (en porcentajes). La primera columna relativa a los que tienen un patrimonio neto mayor a US\$1 millón incorpora a los dos tramos superiores que se presentan en el cuadro. Se tienen coincidencias entre las dos fuentes de información, pero también algunas diferencias. En todo caso Chile es el que tiene el mayor porcentaje de millonarios en todas las categorías respecto del total de la población, le siguen Perú, Brasil, Argentina y Colombia de acuerdo a la data de Credit Suisse. Sin embargo, cuando se utiliza la información de Knight Frank la lista la encabeza México, seguido por Chile, Argentina y Brasil. Por otra parte, las mayores diferencias en las fuentes estadísticas Credit Suisse y Knight Frank se producen en el caso de Perú. En el caso de los que tienen patrimonios netos entre US\$1 y 100 millones la lista liderada por Chile seguido por Brasil y México. De igual forma, el mayor porcentaje de la población que tiene más de US\$100 millones se ubica en Chile, seguido de Perú y Brasil con Credit Suisse. Igualmente, en el caso de Knight Frank, Chile se ubica primero, seguido por México y Brasil.

11 No se presentó la información del promedio mundial de Credit Suisse debido a que se observan inconsistencias en los porcentajes agregados de la riqueza de 10 y $5 \%$ más rico en ambos años. 


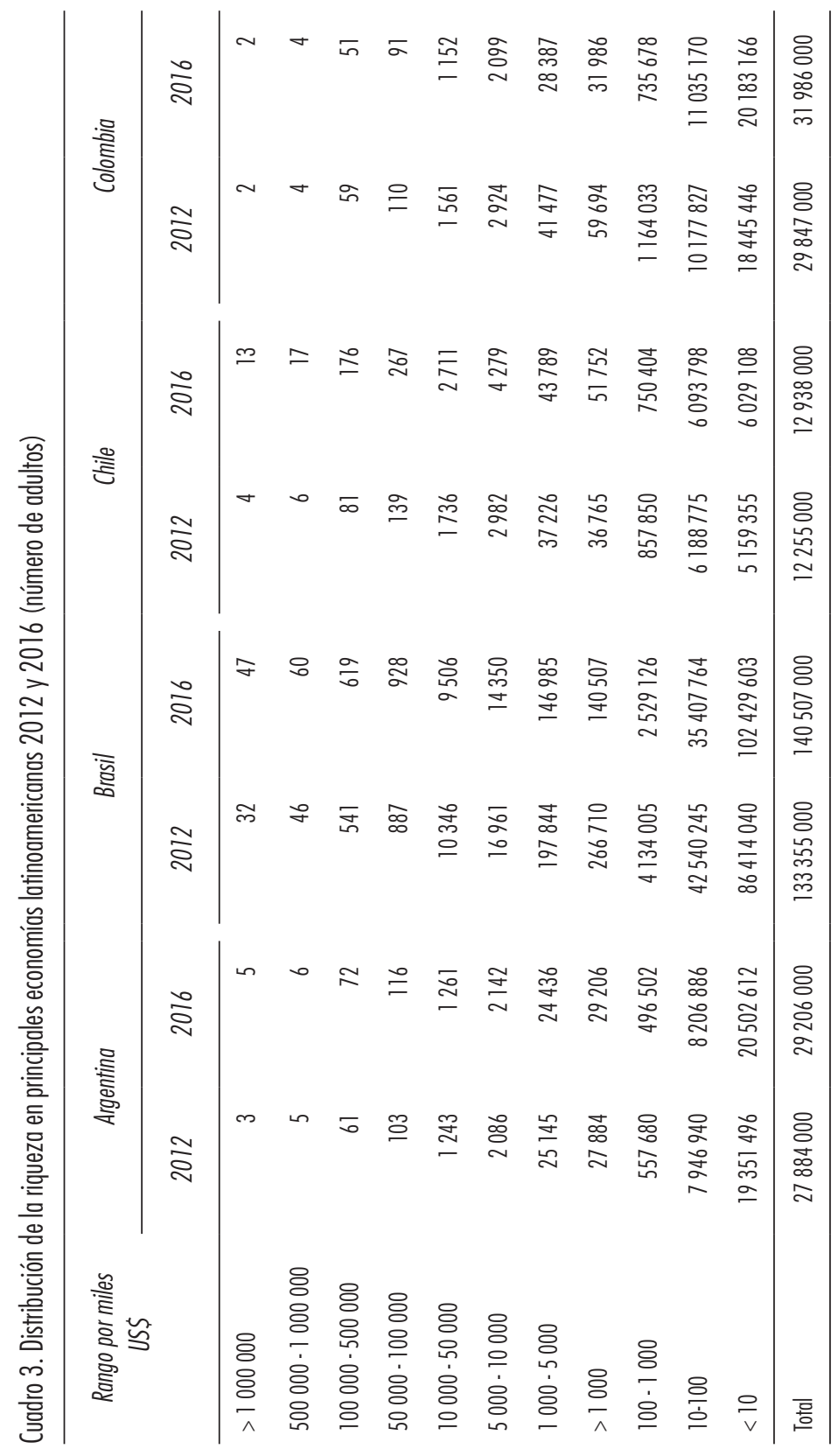




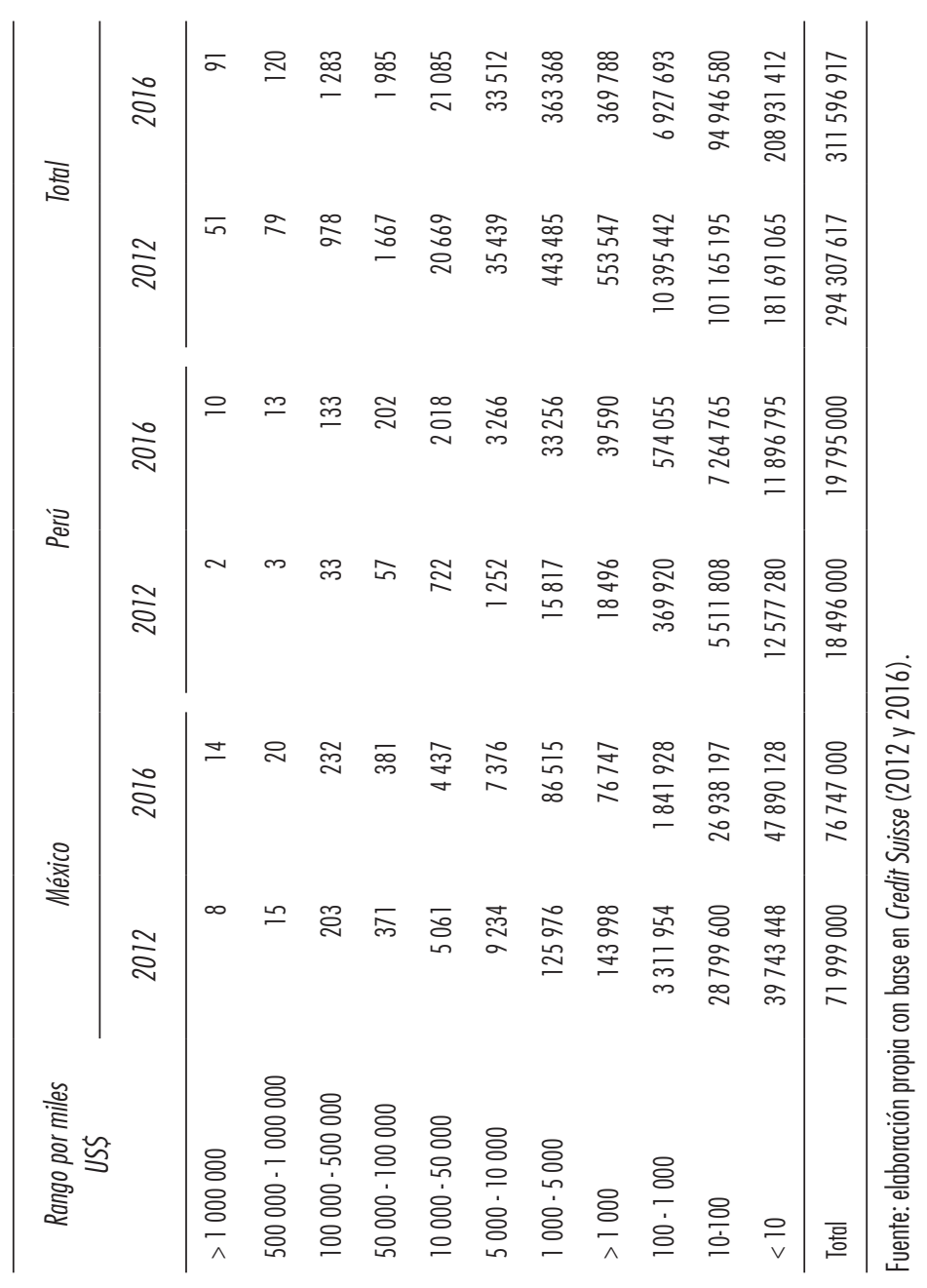




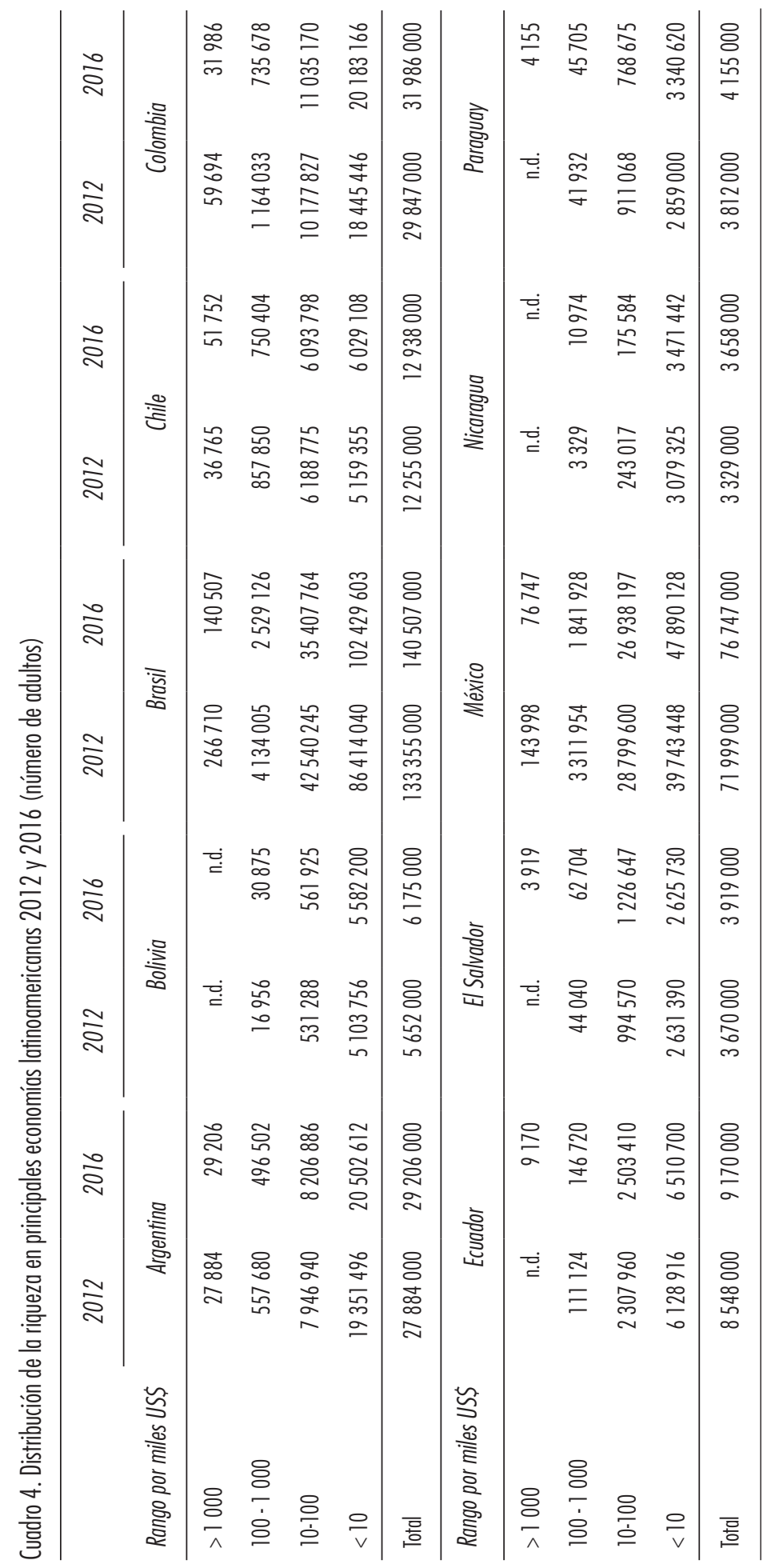




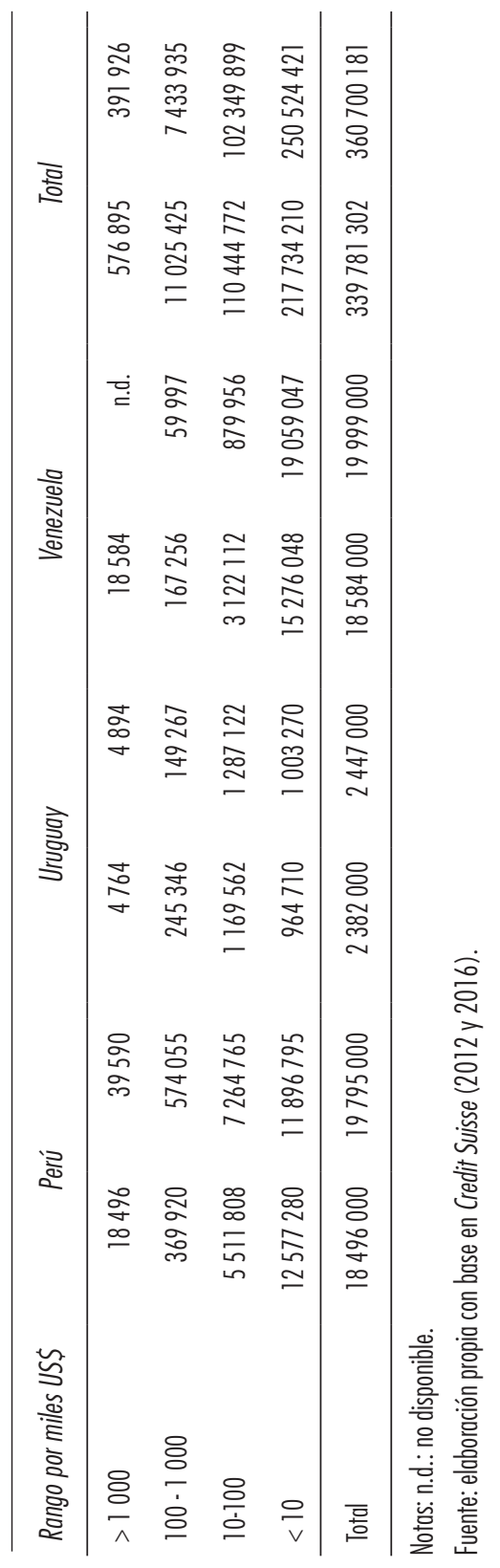


Germán Alarco Tosoni y César Castillo García

Cuadro 5. Riqueza del decil y percentil más rico en economías y regiones predeterminadas 2015-2016 (\%)

\begin{tabular}{|c|c|c|c|c|c|c|}
\hline \multirow[t]{2}{*}{ Economía } & \multicolumn{3}{|c|}{2015} & \multicolumn{3}{|c|}{2016} \\
\hline & $10 \%$ & $5 \%$ & $1 \%$ & $10 \%$ & $5 \%$ & $1 \%$ \\
\hline Brasil & 75.4 & 66.1 & 48.0 & 74.2 & 65.2 & 47.9 \\
\hline Chile & 70.3 & 61.1 & 43.2 & 71.4 & 61.8 & 43.5 \\
\hline Colombia & 66.8 & 54.8 & 34.0 & 63.9 & 52.3 & 32.6 \\
\hline México & 66.0 & 55.2 & 36.0 & 67.1 & 56.8 & 38.2 \\
\hline \multicolumn{7}{|l|}{ Región } \\
\hline África & 78.6 & 68.4 & 47.2 & 82.6 & 72.8 & 51.3 \\
\hline Asia Pacífico & 84.7 & 70.8 & 41.2 & 86.3 & 71.1 & 40.4 \\
\hline China & 65.7 & 56.4 & 39.4 & 73.2 & 63.2 & 43.8 \\
\hline Europa & 70.0 & 55.8 & 32.2 & 71.1 & 56.7 & 32.7 \\
\hline India & 76.3 & 68.6 & 53.0 & 80.7 & 73.4 & 58.4 \\
\hline América Latina & 72.0 & 61.8 & 42.7 & 71.2 & 61.0 & 42.0 \\
\hline América del Norte & 74.3 & 61.8 & 36.7 & 76.0 & 64.8 & 41.0 \\
\hline
\end{tabular}

Fuente: elaboración propia con base en Credit Suisse (2015 y 2016).

Cuadro 6. Distribución de la riqueza en principales economías latinoamericanas 2005, 2015 y 2016 (número de personas)

\begin{tabular}{|c|c|c|c|c|c|c|c|c|c|}
\hline \multirow[t]{2}{*}{ País } & \multicolumn{3}{|c|}{ Entre US\$1-10 millones } & \multicolumn{3}{|c|}{ Entre US\$10-30 millones } & \multicolumn{3}{|c|}{ Entre US\$30-100 millones } \\
\hline & 2005 & 2015 & 2016 & 2005 & 2015 & 2016 & 2005 & 2015 & 2016 \\
\hline Argentina & 21200 & 33500 & 36900 & 920 & 1450 & 1540 & 310 & 500 & 550 \\
\hline Brasil & 101500 & 180000 & 154800 & 5280 & 5200 & 4470 & 2004 & 2400 & 2060 \\
\hline Chile & 9100 & 21200 & 22500 & 490 & 1100 & 1170 & 177 & 320 & 340 \\
\hline Colombia & 25000 & 28200 & 29600 & 940 & 1300 & 1370 & 287 & 450 & 470 \\
\hline México & 128800 & 170000 & 173400 & 5160 & 4800 & 4900 & 1729 & 1900 & 1940 \\
\hline Panamá & 1300 & 3500 & 3700 & 50 & 150 & 160 & 20 & 60 & 60 \\
\hline Paraguay & 700 & 2100 & 2200 & 30 & 90 & 90 & 10 & 30 & 30 \\
\hline Perú & 6100 & 16500 & 17500 & 310 & 830 & 880 & 110 & 280 & 300 \\
\hline Uruguay & 1600 & 4600 & 4800 & 70 & 200 & 210 & 25 & 70 & 70 \\
\hline Venezuela & 1700 & 10500 & 6700 & 70 & 410 & 260 & 26 & 130 & 80 \\
\hline América Latina & 297000 & 470100 & 452100 & 13320 & 15530 & 15050 & 4698 & 6140 & 5900 \\
\hline
\end{tabular}


Cuadro 6. Distribución de la riqueza en principales economías latinoamericanas 2005, 2015 y 2016 (número de personas) (continuación)

\begin{tabular}{|c|c|c|c|c|c|c|}
\hline \multirow[t]{2}{*}{ País } & \multicolumn{3}{|c|}{ Entre US\$100-1 000 millones } & \multicolumn{3}{|c|}{ Más de US\$1 000 millones } \\
\hline & 2005 & 2015 & 2016 & 2005 & 2015 & 2016 \\
\hline Argentina & 35 & 56 & 62 & 3 & 5 & 6 \\
\hline Brasil & 243 & 479 & 407 & 22 & 40 & 34 \\
\hline Chile & 21 & 48 & 51 & 3 & 8 & 6 \\
\hline Colombia & 31 & 50 & 53 & 2 & 3 & 3 \\
\hline México & 191 & 279 & 285 & 12 & 18 & 18 \\
\hline Panamá & 2 & 6 & 6 & 0 & 0 & 0 \\
\hline Paraguay & 1 & 4 & 4 & 0 & 0 & 0 \\
\hline Perú & 13 & 35 & 37 & 2 & 5 & 5 \\
\hline Uruguay & 3 & 8 & 8 & 0 & 0 & 0 \\
\hline Venezuela & 3 & 30 & 19 & 1 & 3 & 2 \\
\hline América Latina & 543 & 995 & 932 & 45 & 82 & 74 \\
\hline
\end{tabular}

Fuente: elaboración propia con base en Knight Frank (2014-2017).

Cuadro 7. Participación de los ricos en el total de la población 2016 (\%)

\begin{tabular}{|c|c|c|c|c|c|c|}
\hline \multirow[t]{2}{*}{ Economías } & \multicolumn{2}{|c|}{ Más de US\$1 millón } & \multicolumn{2}{|c|}{ US\$1-100 millones } & \multicolumn{2}{|c|}{ Más de US\$100 millones } \\
\hline & Credit Suisse & Knight Frank & Credit Suisse & Knight Frank & Credit Suisse & Knight Frank \\
\hline Argentina & 0.06 & 0.08 & 0.06 & n.d & 0.000189 & 0.000141 \\
\hline Brasil & 0.08 & 0.07 & 0.08 & n.d & 0.000350 & 0.000196 \\
\hline Chile & 0.29 & 0.13 & 0.29 & n.d & 0.001150 & 0.000285 \\
\hline Colombia & 0.07 & 0.06 & 0.07 & n.d & 0.000117 & 0.000109 \\
\hline México & 0.08 & 0.14 & 0.08 & n.d & 0.000209 & 0.000223 \\
\hline Perú & 0.12 & 0.06 & 0.12 & n.d & 0.000491 & 0.000116 \\
\hline América Latina & 0.09 & 0.09 & 0.09 & n.d & 0.000313 & 0.000187 \\
\hline
\end{tabular}

Notas: n.d.: no disponible.

Fuente: elaboración propia con base en Knight Frank (2017), Credit Suisse (2016) y Banco Mundial (2018b). 
En el cuadro 8 se presenta la concentración de la riqueza de las principales economías de América Latina. Se muestra el coeficiente Gini calculado por Credit Suisse para cada economía en particular, mientras que el regional se recalcula de acuerdo a un promedio ponderado del PIB de paridad de poder adquisitivo para el periodo 2010-2016 en US\$ constantes con base 2011 reportados por el Banco Mundial. En el caso de Venezuela los ańos 2015 y 2016 se ajustaron por la caída en el producto real de 2015 y 2016 del Fondo Monetario Internacional (FMI).

Cuadro 8. Concentración de riqueza en principales economías de América Latina 2010-2016 (Gini)

\begin{tabular}{llllllll}
\hline País & 2010 & 2011 & 2012 & 2013 & 2014 & 2015 & 2016 \\
\hline Argentina & 0.747 & 0.768 & 0.782 & 0.796 & 0.809 & 0.818 & 0.787 \\
Bolivia & 0.773 & 0.756 & 0.745 & 0.744 & 0.745 & 0.713 & 0.779 \\
Brasil & 0.796 & 0.795 & 0.812 & 0.821 & 0.823 & 0.830 & 0.829 \\
Chile & 0.647 & 0.782 & 0.774 & 0.814 & 0.789 & 0.795 & 0.805 \\
Colombia & 0.795 & 0.792 & 0.788 & 0.797 & 0.768 & 0.769 & 0.762 \\
Costa Rica & 0.767 & 0.721 & 0.723 & 0.722 & 0.767 & 0.814 & 0.734 \\
Ecuador & 0.768 & 0.744 & 0.715 & 0.714 & 0.752 & 0.738 & 0.756 \\
El Salvador & 0.733 & 0.710 & 0.711 & 0.710 & 0.726 & 0.707 & 0.726 \\
México & 0.780 & 0.774 & 0.780 & 0.780 & 0.759 & 0.759 & 0.779 \\
Nicaragua & 0.752 & 0.735 & 0.730 & 0.730 & 0.714 & 0.672 & 0.767 \\
Panamá & 0.795 & 0.743 & 0.727 & 0.727 & 0.783 & 0.802 & 0.766 \\
Paraguay & 0.756 & 0.738 & 0.728 & 0.728 & 0.756 & 0.729 & 0.774 \\
Perú & 0.752 & 0.725 & 0.774 & 0.708 & 0.817 & 0.803 & 0.807 \\
Uruguay & 0.810 & 0.710 & 0.698 & 0.698 & 0.777 & 0.825 & 0.699 \\
Venezuela & 0.720 & 0.806 & 0.796 & 0.825 & 0.818 & 0.818 & 0.837 \\
América Latina & 0.772 & 0.780 & 0.789 & 0.795 & 0.795 & 0.798 & 0.799 \\
\hline
\end{tabular}

Fuente: elaboración propia con base en Credit Suisse (2011-2016), Banco Mundial (2018a) y FMI (2018).

En todos los casos, los coeficientes Gini son elevados reflejando una elevada concentración. Las economías de mayores niveles de concentración de la riqueza a 2016 son Venezuela, Brasil, Perú y Chile; mientras que las de me- 
nor nivel de concentración son Uruguay, El Salvador, Costa Rica, Ecuador, Colombia, Panamá y Nicaragua. A un nivel intermedio se ubican Argentina, Bolivia, México y Paraguay. Se observan tendencias crecientes claras en las series de Brasil y Chile. Existe una tendencia creciente pero fluctuante en Argentina, Perú y Venezuela. En el resto de las economías como Bolivia, Colombia, Costa Rica, Ecuador, El Salvador, México, Nicaragua, Panamá, Paraguay y Uruguay se observan mayor variabilidad. En el promedio ponderado de las diferentes economías de la región se observa una tendencia ascendente clara desde un valor de 0.77 en 2010 hasta casi 0.8 en 2016. En lo que va del segundo quinquenio del siglo XXI la concentración de la riqueza muestra una tendencia creciente.

\section{COMPARACIONES INTERREGIONALES}

Es útil contrastar la información de los niveles de concentración de América Latina respecto de las otras regiones y el promedio mundial. Para tal efecto se empleó la información regional procesada por Credit Suisse y el promedio mundial obtenido de acuerdo a la ponderación de cada una de las regiones en el рів de paridad de poder adquisitivo en US\$ de 2011 en el mundo. En el cuadro 9 se presentan las regiones analizadas tomando en consideración América Latina y el Caribe del Credit Suisse que incluye a las naciones que no son hispano hablantes y del Caribe. Asimismo, se muestran los resultados de América Latina incluyendo las 15 economías consideradas. De igual forma que en el cuadro 5 hay que destacar que todos los niveles de concentración son elevados.

En 2010 los niveles más elevados de concentración se encontraban en la región Asia Pacífico seguido por África. Mientras que China y América Latina se ubicaban entre las regiones con menores niveles de concentración que el resto de las regiones. Para 2016 los mayores niveles de concentración del mundo se localizaban en la región Asia Pacífico seguidos por África, India, América del Norte y Europa. Los más bajos se observaban en América Latina y China. En general casi todas las regiones tienen niveles de concentración crecientes destacando América Latina, Asia Pacífico, China, Europa e India, seguidos por África y América del Norte. El promedio mundial del coeficiente Gini del mundo tiene también una tendencia a la alza al pasar de casi 0.8 en 2010 a 0.86 en 2016. La riqueza tiene a concentrarse más en pocas manos al igual que la tendencia en la concentración de los ingresos (Piketty, 2019). 
Germán Alarco Tosoni y César Castillo García

Cuadro 9. Concentración de riqueza en principales regiones del mundo 2010-2016 (Gini)

\begin{tabular}{llllllll}
\hline Regiones & 2010 & 2011 & 2012 & 2013 & 2014 & 2015 & 2016 \\
\hline África & 0.849 & 0.872 & 0.865 & 0.846 & 0.856 & 0.856 & 0.887 \\
América del Norte & 0.799 & 0.816 & 0.842 & 0.841 & 0.837 & 0.842 & 0.852 \\
América Latina y Caribe & 0.785 & 0.793 & 0.797 & 0.806 & 0.809 & 0.809 & 0.813 \\
América Latina & 0.772 & 0.780 & 0.789 & 0.795 & 0.795 & 0.798 & 0.799 \\
Asia Paćfico & 0.869 & 0.881 & 0.889 & 0.887 & 0.895 & 0.892 & 0.904 \\
China & 0.690 & 0.697 & 0.689 & 0.695 & 0.719 & 0.733 & 0.819 \\
Europa & 0.799 & 0.829 & 0.831 & 0.830 & 0.827 & 0.834 & 0.849 \\
India & 0.778 & 0.804 & 0.813 & 0.813 & 0.814 & 0.831 & 0.876 \\
Mundo & 0.799 & 0.816 & 0.822 & 0.821 & 0.825 & 0.830 & 0.857 \\
\hline
\end{tabular}

Fuente: elaboración propia con base en Credit Suisse (2010-2016), Banco Mundial (2018a) y FMI (2018).

\section{CONCLUSIONES}

El estudio de los niveles de riqueza y su concentración es complejo ante la inexistencia de información oficial sobre el tema de la mayor parte de las economías de América Latina. Es pues urgente que desde la academia y la sociedad civil se presione a los respectivos gobiernos para que elaboren y transparenten la información sobre la materia. Por el momento, la alternativa se encuentra en el uso de fuentes independientes, pero con el problema de que cada una de ellas tiene una metodología particular, mostrando resultados diferenciados entre sí. Las estimaciones de la riqueza productiva del Banco Mundial se realizan a partir de los valores contables agregados de la formación bruta de capital. Estos resultados divergen de los criterios de valorización de acuerdo a mercado de las fuentes independientes y, por tanto, sujetos a numerosas variables económicas, financieras, sociales y políticas.

A pesar de las diferencias entre las fuentes de información y las limitadas series estadísticas se extraen tendencias comunes. En la segunda década del siglo Xxi la concentración de la riqueza tiene una tendencia creciente; lo que va en la misma línea de lo señalado en diversos estudios internacionales sobre dicha problemática, especialmente en lo relativo a la concentración de los ingresos. Sin embargo, la evidencia disponible no permite evaluar lo ocurrido en la región antes y durante la edad de oro del capitalismo. 
Los superricos han incrementado su número e importancia tanto a nivel global como regional en América Latina. Se tiene una clara tendencia ascendente con relación a sus respectivos pIB en lo que va del siglo XXI. Tienen mayor presencia absoluta en Brasil, pero relativamente respecto a la población es mayor en Chile. Asimismo, las personas que tienen riquezas superiores a US $\$ 50$ millones, para el conjunto de economías de la región, se han incrementado significativamente; mientras que los que tienen entre US\$5 y 50 millones mantienen una situación estacionaria y el número de millonarios entre US\$1 y 5 millones se redujo por las caídas de Argentina, Brasil, Colombia y México. Con la información de Knight Frank el número de las personas en América Latina que tienen entre US\$1 y 10 millones se redujo entre 2005 y 2016. Sólo Chile y Perú muestran aumentos en esta categoría de pequeños millonarios.

El 1\% de la población más rica de América Latina mantiene 42\% de la riqueza con una mayor participación que en las regiones europeas, Asia Pacífico y ligeramente superior a América del Norte. Por otra parte, el número de las personas que tienen un patrimonio neto menor a US\$10 000 se incrementó significativamente; mientras que se redujo el relativo a los que tienen entre US $\$ 10000$ y 1 millón por lo que se vislumbra una caída en los patrimonios de los estratos medios y medios-altos entre 2012 y 2016 con algunas excepciones.

Los coeficientes Gini de concentración de la riqueza de toda América Latina tienen una tendencia creciente entre 2010-2016. Al interior de la región Brasil, Argentina, Chile, Perú y Venezuela tienen la misma trayectoria ascendente. En 2016 Venezuela, Brasil, Perú y Chile fueron las economías con los mayores niveles de concentración. El coeficiente Gini del mundo también presenta una tendencia creciente. Con la información obtenida de Credit Suisse, América Latina tiene una concentración de la riqueza muy alta y creciente, pero inferior al resto de regiones del mundo.

El hecho de que América Latina no sea la región con los mayores niveles de concentración de la riqueza del mundo, no significa un gran consuelo, ya que los coeficientes Gini son extremadamente elevados, mismos que condicionarían una distribución factorial y funcional del ingreso a favor de las ganancias y una elevada concentración en la distribución personal del ingreso. A diferencia de las economías más desarrolladas, en la región de América Latina el rol redistributivo de la política fiscal es menor. Los impuestos a las herencias son entre inexistentes, muy pequeños o recientes. Por esta razón las posibilidades de que la distribución personal del ingreso sea más equitativa son reducidas. 


\section{BIBLIOGRAFÍA}

Alarco, G. (2014), "Participación salarial y crecimiento económico en América Latina 1950-2011”, Revista CEPAL, núm. 113. Disponible en <https:// www.cepal.org/es/publicaciones/36958-participacion-salarial-crecimiento-economico-america-latina-1950-2011>

(2017), "Ciclos distributivos y crecimiento económico en América Latina”, Cuadernos de Economía, vol. 36, núm. 72, DoI <10.15446/cuad. econ.v36n72.65819>

(2018), "Mergers and acquisitions in Latin America 1990-2014: factorial distribution and contractionary impacts", Metroeconomica, vol. 69, núm. 3, DOI <https://doi.org/10.1111/meca.12208>

Alarco, G., Castillo, C. y Leiva, F. (2019), Riqueza y desigualdad en el Perú: visión panorámica, Lima, oxfaM.

Alvaredo, F. y Gasparini, L. (2015), "Recent trends in inequality and poverty in developing countries", Handbook of Income Distribution, Amsterdam, Elsevier.

Alvaredo, F., Chancel, L., Piketty, T., Saez, E. y Zucman, G. (2019), Informe sobre la desigualdad global 2018, México, Libros Grano de Sal.

Báez Melián, J. (2018), "Los efectos de la política fiscal sobre la desigualdad", Revista de Economía Mundial, núm. 49, DOI <http://dx.doi.org/10.33776/ rem.v0i49.3890>

Banco Mundial (2011), The changing wealth of nations 2011, Washington, D.C., The World Bank.

(2018a), The changing wealth of nations 2018: Building a sustainable future, Washington, D.C., The World Bank.

(2018b), World development indicators, <http://databank.bancomundial.org/data/reports.aspx?source=2\&country=PER>

Bourguignon, F. [2017 (2012)], La globalización de la desigualdad, México, Fondo de Cultura Económica.

CEPAL (2018), La ineficiencia de la desigualdad 2018. Trigésimo séptimo periodo de sesiones de la CEPAL, Santiago, Naciones Unidas.

Credit Suisse (2011-2017), Global Wealth Databook 2010, Zurich, Switzerland.

Davies, J. y Shorrocks, A. (2000), "The distribution of wealth", en A. Atkinson y F. Bourguignon (eds.), Handbook of Income Distribution, vol. 1, Holanda, North Holland. 
, Sandström, S., Shorrocks, A. y Wolff, E. (2010), "The level and distribution of global household wealth", The Economic Journal, vol. 121, núm. 551, DOI <https://doi.org/10.1111/j.1468-0297.2010.02391.x>

De Rosa, M. (2019), Wealth accumulation and its distribution in Uruguay: First estimates of the untold half of story. Public Policies and Development Master Thesis, Paris, Paris School of Economics.

Fondo Monetario Internacional (FMI) (2018), International Financial Statistics, International Monetary Fund. Disponible en <https://data.imf. org/?sk=4c514d48-b6ba-49ed-8ab9-52b0c1a0179b>

Forbes (2010-2017), The Forbes world's billionaires list, Forbes, Nueva York, Forbes.

Foro Económico Mundial (2018), The global risks report 2018, Geneve, FEM. Giffen, R. (1913), Statistics, Macmillan, Londres.

Goldsmith, R. (1962), The national wealth of the United States in the postwar period, New Jersey, Princenton University Press.

Jiménez, J. (2015), Desigualdad, concentración del ingreso y tributación sobre las altas rentas en América Latina, Santiago, CEPAL.

Knight, F. (2014-2017), The wealth report: The global perspective on prime property and investment, London, Knight Frank Research.

Lustig, N. y López-Calva, L. (2010), Declining inequality in Latin America, a decade of progress?, Washington, D.C., Brookings Institute.

Milanovic, B. [2017 (2016)], Desigualdad mundial. Un nuevo enfoque para la era de la globalización, México, Fondo de Cultura Económica.

Organización para la Cooperación y el Desarrollo Económicos (OCDE) (2008), Growing unequal? Income distribution and poverty in OECD countries, Paris, OCDE.

Ostry, J., Berg, A. y Tsangarides, Ch. (2014), Redistribution, inequality, and growth (IMF Staff Discussion Note SDN/14/02). http://www.imf.org/external/ns/search.aspx?hdCountrypage $=\& N$ ewQuery $=$ ostry+berg+tsangar ides\&search $=$ Search\&filter_val=N\&col=SITENG\&collection=SITENG \&lan $=$ eng $\&$ iso $=\&$ requestfrom $=\&$ countryname $=\& \mathrm{f}=$

oxfam (2016), Una economía al servicio del 1\%. Informe de oxfam 210, Oxford.

Piketty, T. (2014), El capital en el siglo XXI, México, Fondo de Cultura Económica.

(2019), Capital e ideología, Colombia, Editorial Planeta.

Piketty, T. y Saez, E. (2003), "Income inequality in the United States 19131998", The Quaterly Journal of Economic, vol. 118, núm. 1, Oxford Academic. 
Roine, J. y Waldenström, D. (2015), "Long-run trends in the distribution of income and wealth", en A. Atkinson y F. Bourguignon (eds.), Handbook of Income Distribution, vol. 2A, Holanda, North Holland.

Saez, E. y Zucman, G. (2016), "Wealth inequality in the United States since 1913: Evidence from capitalized income tax data", The Quarterly Journal of Economics, vol. 131, núm. 2. Disponible en <http://qje.oxfordjournals. org/>

Solimano, A. (2016), "Is inequality really declining in Latin America? Evidence on income, wealth and the social structure", Revista de Economia Mundial, núm. 43, DOI <http://dx.doi.org/10.33776/rem.v0i43.3859>

Trueba, C. y Remuzgo, L. (2016), Evolution of inequality in Latin America (1980-2014), Revista de Economía Mundial, 45, España, Universidad de Huelva. Disponible en <http://rabida.uhu.es/dspace/bitstream/handle/10 272/13731/Evolution_of_inequality\%20.pdf?sequence=2> 